\title{
PRIMER NOTE \\ Isolation and characterization of microsatellite markers in Fraser fir (Abies fraseri)
}

\author{
S. A. JOSSERAND,${ }^{*}$ K. M. POTTER,, G. JOHNSON,${ }^{*}$ J. A. BOWEN,+ J. FRAMPTON $\ddagger$ and \\ C. D. NELSON* \\ *USDA Forest Service, Southern Institute of Forest Genetics, 23332 Mississippi 67, Saucier, MS 39574, USA, +University of Southern \\ Mississippi, Department of Biological Sciences, Gulf Park Campus, Long Beach, MS 39560, USA, †North Carolina State University, \\ Department of Forestry and Environmental Resources, Raleigh, NC 27695, USA
}

\begin{abstract}
We describe the isolation and characterization of 14 microsatellite loci from Fraser fir (Abies fraseri). These markers originated from cloned inserts enriched for DNA sequences containing tandem di- and tri-nucleotide repeats. In total, 36 clones were selected, sequenced and evaluated. Polymerase chain reaction (PCR) primers for 14 of these sequences consistently produced simple PCR profiles and were found to be polymorphic among 13 Fraser fir samples. In addition, more than half of these loci were found to amplify a wide range of samples from several Abies taxa.
\end{abstract}

Keywords: conservation genetics, fir, microsatellite DNA

Received 27 April 2005; revision received 18 June 2005; revision accepted 18 July 2005

The true firs (Abies) comprise more than 45 species discontinuously distributed in the Northern Hemisphere and are most frequently occurring in montane to subapline zones of mountain systems. Classification of these species (and dozens of varieties) is complicated by the existence of closely related sibling species and the hybridization of species with overlapping ranges (Liu 1971; Farjon \& Rushforth 1989). Fraser fir [Abies fraseri (Pursh) Poir.], a species endemic to a handful of high-elevation ridge systems in the southern Appalachian Mountains of the United States, has suffered dramatic mortality over the past half century as a result of infestation by the exotic balsam woolly adelgid (Adelges piceae Ratz.) (Dull et al. 1988). Fraser fir faces additional adelgid mortality and changing climate conditions that could make its existing habitat unsuitable for long-term viability (Nicholas et al. 1999). Efforts to conserve the genetic diversity of this ecologically and economically important species, which is the basis of the $\$ 100$ million annual Christmas tree industry in North Carolina, require a better understanding of the genetic architecture of its populations. Microsatellite DNA markers are considered to be highly informative for assessing genetic diversity and population structure. Here we present results from a project to develop

Correspondence: C. D. Nelson, Fax: 228-832-0130;

E-mail: dananelson@fs.fed.us microsatellite markers from Fraser fir for use in Fraser fir and other Abies taxa.

Fraser fir genomic DNA was partially restricted with a cocktail of seven blunt-end cutting enzymes (RsaI, HaeIII, BsrB1, PvuII, StuI, ScaI and EcoRV). Fragments in the size range of 300-750 bp were adapted and subjected to magnetic bead capture (CPG, Inc.), using biotinylated capture molecules. Libraries were prepared in parallel using biotin-(CA) ${ }_{15}$ (AC library), biotin-(GA) ${ }_{15}$ (AG library), biotin$\mathrm{AAT}_{15}$ (AAT library) and biotin-(TAGA) 8 (AGAT library) as capture molecules in a protocol provided by the manufacturer. Captured molecules were amplified and restricted with HindIII to remove the adapters. The resulting fragments were ligated into pUC19 and recombinant molecules were electroporated into Escherichia coli $\mathrm{DH} 5 \alpha$. Thirty-six recombinant colonies (nine each from the AC, AG, AAT and AGAT libraries) were selected at random and sequenced on an ABI 377 using ABI PRISM Taq dye terminator cycle sequencing methodology (Applied Biosystems). Relatively long repeats that were centrally located in the sequence were identified for 20 of these inserts, nine each from the AC and AG libraries and two from the AAT library. Polymerase chain reaction (PCR) primer pairs were designed for each of these inserts using PRIMER 3.0 (Rozen \& Skaletsky 1996) and synthesized (Integrated DNA Technologies; Applied Biosystems) for further evaluation (Table 1). All 
Table 1 Characterization of 14 microsatellite loci isolated from Fraser fir (Abies fraseri)

\begin{tabular}{|c|c|c|c|c|c|c|c|c|c|}
\hline $\begin{array}{l}\text { Locus } \\
\text { name }\end{array}$ & $\begin{array}{l}\text { GenBank } \\
\text { Accession and } \\
\text { dbSTS ID nos }\end{array}$ & $\begin{array}{l}\text { Repeat } \\
\text { motif } \\
\text { class }\end{array}$ & Observed repeat & Primer sequences $\left(5^{\prime}-3^{\prime}\right)$ & $\begin{array}{l}\text { PCR } \\
\text { protocol }\end{array}$ & $\begin{array}{l}\text { Number of } \\
\text { alleles: range } \\
\text { in size (bp) }\end{array}$ & $H_{\mathrm{O}}$ & $H_{\mathrm{E}}$ & $\begin{array}{l}\text { LR test } \\
\text { of HWE }\end{array}$ \\
\hline AfSI_02 & $\begin{array}{l}\text { BV677350 } \\
808313\end{array}$ & $\mathrm{AC}$ & $(\mathrm{AT})_{10}(\mathrm{C})(\mathrm{GT})_{12}(\mathrm{AT})_{2}$ & $\begin{array}{l}\text { CCTAGTGAGGCATTGTATAGACCC } \\
\text { TAGATATCTGCAGGTCCGCC }\end{array}$ & 3 & $6: 361-387$ & 0.33 & 0.72 & 0.23 \\
\hline AfSI_03 & $\begin{array}{l}\text { BV677351 } \\
808314\end{array}$ & $\mathrm{AC}$ & $(\mathrm{AT})_{10}(\mathrm{C})(\mathrm{GT})_{12}(\mathrm{AT})_{2}$ & $\begin{array}{l}\text { GATCATGAAGCTCACCAACATC } \\
\text { ACATGGGGAGATTTTTTGCAG }\end{array}$ & 1 & 2: $255-259$ & 0.46 & 0.47 & 0.93 \\
\hline AfSI_04 & $\begin{array}{l}\text { BV677345 } \\
808308\end{array}$ & $\mathrm{AC}$ & $(\mathrm{AC})_{36}$ & $\begin{array}{l}\text { ATTCCAGAGGGGTCATTGC } \\
\text { ACAGTGTGTCGCTGAAGTGC }\end{array}$ & 2 & 2: $176-179$ & 1.0 & 0.50 & 0.00 \\
\hline AfSI_05 & $\begin{array}{l}\text { BV677346 } \\
808309\end{array}$ & $\mathrm{AC}$ & $(\mathrm{AC})_{3}(\mathrm{AA})(\mathrm{AC})_{15}(\mathrm{AT})_{4}(\mathrm{AC})(\mathrm{AT})$ & $\begin{array}{l}\text { CGAAGGACTCGTGACAGTGA } \\
\text { CATGTGTAAATAAAATGCGTCAAA }\end{array}$ & 1 & $3: 321-333$ & 0.17 & 0.47 & 0.05 \\
\hline AfSI_06 & $\begin{array}{l}\text { BV677347 } \\
808310\end{array}$ & $\mathrm{AC}$ & $(\mathrm{AC})_{24}$ & $\begin{array}{l}\text { CAAACTGAGGATGGGTATTGG } \\
\text { ATTGTGTGGTTCATCCCACC }\end{array}$ & 2 & $4: 337-346$ & 0.58 & 0.55 & 0.51 \\
\hline AfSI_07 & $\begin{array}{l}\text { BV677348 } \\
808311\end{array}$ & $\mathrm{AC}$ & $(\mathrm{CT})_{2}(\mathrm{GT})_{14}$ & $\begin{array}{l}\text { GTCACATTATGCCTCTCCCC } \\
\text { CTTACCACCACCAATGTGGG }\end{array}$ & 2 & $3: 255-278$ & 0.39 & 0.32 & 0.75 \\
\hline AfSI_08 & $\begin{array}{l}\text { BV677349 } \\
808312\end{array}$ & $\mathrm{AC}$ & $(\mathrm{GT})_{19}$ & $\begin{array}{l}\text { GATGATGGTCTTGTGTCTGC } \\
\text { TATCACTGGTAGTGGATTTCA }\end{array}$ & 1 & 5: 198-206 & 0.69 & 0.66 & 0.69 \\
\hline AfSI_11 & $\begin{array}{l}\text { BV677356 } \\
808319\end{array}$ & AG & $(\mathrm{AT})_{2}(\mathrm{AC})_{4}(\mathrm{AG})_{27}$ & $\begin{array}{l}\text { TGCAGCCCCCTATTAGTCAC } \\
\text { TTGTGTATTTTGTATTGTATTGCTCTG }\end{array}$ & 2 & 13: $233-263$ & 1.0 & 0.90 & 1.0 \\
\hline AfSI_14 & $\begin{array}{l}\text { BV677352 } \\
808315\end{array}$ & $\mathrm{AG}$ & $(\mathrm{AG})_{7} \ldots(\mathrm{GA})_{24}$ & $\begin{array}{l}\text { ATGGGAGTGCAAAATCAACC } \\
\text { CACCCCAAACAGATCCAAAG }\end{array}$ & 2 & 5: 191-230 & 0.39 & 0.39 & 0.96 \\
\hline AfSI_15 & $\begin{array}{l}\text { BV677353 } \\
808316\end{array}$ & AG & $(\mathrm{AG})_{18}$ & $\begin{array}{l}\text { ATGGATGCGAGAGAACATCC } \\
\text { TCAACTAACTGGAGTCCCCC }\end{array}$ & 1 & 14: $281-334$ & 0.85 & 0.91 & 1.0 \\
\hline AfSI_16 & $\begin{array}{l}\text { BV677354 } \\
808317\end{array}$ & AG & $(\mathrm{CT})_{14}$ & $\begin{array}{l}\text { CTATGGCTGCATCAATTGCC } \\
\text { ATAATCCACGAGTCGATGCC }\end{array}$ & 2 & 8: 289-308 & 0.92 & 0.81 & 0.95 \\
\hline AfSI_18 & $\begin{array}{l}\text { BV677355 } \\
808318\end{array}$ & $\mathrm{AG}$ & $(\mathrm{TC})_{19}(\mathrm{TA})_{10}$ & $\begin{array}{l}\text { ATAAGCAACTTGGTGGGCAG } \\
\text { TCACATTATAAACTCAGTTGTGCG }\end{array}$ & 1 & $9: 235-270$ & 0.85 & 0.86 & 0.91 \\
\hline AfSI_19 & $\begin{array}{l}\text { BV677356 } \\
808319\end{array}$ & $\mathrm{AAT}$ & $(\mathrm{CCT})_{3}(\mathrm{CCA} \ldots \mathrm{TAT})(\mathrm{CCT})_{2}$ & $\begin{array}{l}\text { AGGGTGCAGTAGGAAGAGGG } \\
\text { TAGTGGGTTGGTTGGGAGAG }\end{array}$ & 1 & $4: 348-362$ & 0.69 & 0.67 & 0.47 \\
\hline AfSI_20 & $\begin{array}{l}\text { BV677358 } \\
808321\end{array}$ & $\mathrm{AAT}$ & $(\mathrm{GTT})_{2}(\mathrm{AAT})_{9}$ & $\begin{array}{l}\text { CATTATGTTGGCTAGTAAGGTCAC } \\
\text { CCTACTCATTCGATGGGCTC }\end{array}$ & 1 & $2: 308-311$ & 1.0 & 0.50 & 0.00 \\
\hline
\end{tabular}

Notes: sequences will be available on GenBank on 1 August 2005. PCR protocol: 1, standard (see text); 2, standard with hot start Taq DNA polymerase; and 3, standard with Hot Start Taq and $1 \mathrm{mM} \mathrm{MgCl}_{2}$. Number of alleles and minimum-to-maximum allele size (bp) observed in the Fraser fir sample $(n=13)$ scored with GENESCAN/GENOTYPER software. $H_{\mathrm{O}}$, observed heterozygosity; $H_{\mathrm{E}}$, expected heterozygosity (assuming Hardy-Weinberg equilibrium, HWE); LR test, $P$-values for likelihood ratio tests of HWE (Weir 1990), programmed using SAS (1990). 
Table 2 Across-Abies taxa allele amplification of 14 microsatellite markers isolated from Fraser fir (Abies fraseri)

\begin{tabular}{|c|c|c|c|c|c|c|c|c|c|c|c|c|c|c|c|}
\hline Abies species & Seed source & $\begin{array}{c}\text { AfSI } \\
-02\end{array}$ & $\begin{array}{c}\text { AfSI } \\
-03\end{array}$ & $\begin{array}{c}\text { AfSI } \\
-04\end{array}$ & $\begin{array}{c}\text { AfSI } \\
-05\end{array}$ & $\begin{array}{c}\text { AfSI } \\
-06\end{array}$ & $\begin{array}{c}\text { AfSI } \\
-07\end{array}$ & $\begin{array}{c}\text { AfSI } \\
-08\end{array}$ & $\begin{array}{l}\text { AfSI } \\
\_11\end{array}$ & $\begin{array}{c}\text { AfSI } \\
\_14\end{array}$ & $\begin{array}{l}\text { AfSI } \\
\_15\end{array}$ & $\begin{array}{l}\text { AfSI } \\
-16\end{array}$ & $\begin{array}{c}\text { AfSI } \\
\_18\end{array}$ & $\begin{array}{l}\text { AfSI } \\
\_19\end{array}$ & $\begin{array}{c}\text { AfSI } \\
\_20\end{array}$ \\
\hline A. fraseri & North Carolina, USA & + & + & + & + & + & + & + & + & + & + & + & + & + & + \\
\hline A. balsamea & var. 'New England', USA & + & + & + & + & + & + & + & + & + & - & + & + & + & + \\
\hline A. sachalinensis & Unknown, Japan & - & + & + & + & + & + & - & - & + & - & + & + & + & + \\
\hline A. concolor & Rocky Mountains, USA & - & + & + & - & + & + & + & - & + & - & + & + & - & - \\
\hline A. sibirica & Unknown, Russia & - & + & + & + & + & + & + & + & + & - & + & - & - & - \\
\hline A. religiosa & Unknown, Mexico & - & + & + & - & + & + & + & - & + & - & + & - & - & - \\
\hline A. grandis & Pacific Coast, USA & - & + & + & - & - & + & + & - & + & - & + & + & - & - \\
\hline A. alba & Unknown, Europe & - & - & + & - & + & - & + & - & + & - & + & + & + & - \\
\hline
\end{tabular}

Note: species are sorted top to bottom from most (14) to least (7) markers amplifying. +, successful amplification;-, unsuccessful amplification.

forward primers were $5^{\prime}$ end-labelled with one of the following fluorophores: 6-FAM, VIC, PET or NED.

The 20 primer pairs were screened with genomic DNA samples from 13 Fraser fir trees (representing the six major populations) and 3-tree pools of genomic DNA from seven additional Abies taxa using the following PCR protocol: $10 \mathrm{ng}$ genomic DNA, $200 \mu \mathrm{M}$ each primer, $200 \mu \mathrm{M}$ dNTPs, $1 \times$ Taq buffer (2.0 mM $\mathrm{MgCl}_{2}, 10 \mathrm{~mm}$ Tris-Cl, $50 \mathrm{~mm} \mathrm{KCl}$ ) and $0.5 \mathrm{U}$ Taq DNA polymerase (Promega) in $12 \mu \mathrm{L}$ total volume. The PCRs were completed using the following touchdown protocol on PTC-100 thermal cyclers (MJ Research): $2 \mathrm{~min}$ at $94^{\circ} \mathrm{C}$; followed by 20 cycles of $30 \mathrm{~s}$ at $94{ }^{\circ} \mathrm{C}, 30 \mathrm{~s}$ at $\mathrm{X}$, and $30 \mathrm{~s}$ at $72{ }^{\circ} \mathrm{C}$ where $\mathrm{X}=65^{\circ} \mathrm{C}$ in the first cycle decreasing by $0.5^{\circ} \mathrm{C}$ every cycle thereafter; followed by 15 cycles of $30 \mathrm{~s}$ at $92{ }^{\circ} \mathrm{C}, 30 \mathrm{~s}$ at $55^{\circ} \mathrm{C}, 1 \mathrm{~min}$ at $72{ }^{\circ} \mathrm{C}$; followed by a $15 \mathrm{~min}$ extension at $72{ }^{\circ} \mathrm{C}$ and an indefinite hold at $4{ }^{\circ} \mathrm{C}$. The resulting PCR products were separated on an ABI PRISM 3100 Genetic Analyser (Applied Biosystems) as recommended by the manufacturer. Peaks were sized (base pairs) and alleles were scored using GENESCAN and GENOTYPER softwares (Applied Biosystems), respectively, using GS (500-250) LIZ as an internal size standard. Amplification of some primer pairs were markedly improved with a Taq DNA polymerase designed for Hot Start PCR and/or modifications of $\mathrm{MgCl}_{2}$ concentration (Table 1).

Eighteen of the 20 primer pairs successfully amplified products at or near the expected size (data not shown). Among the Fraser fir samples, 15 primer pairs provided interpretable allele data and 14 of these were polymorphic (Table 1). Recommended modifications of the PCR protocol for each marker locus are provided in Table 1 . The 14 polymorphic loci had from two to 14 alleles (average $=5.7$ ) across the 13 Fraser fir samples with expected heterozygosities $\left(H_{\mathrm{E}}\right)$ ranging from 0.32 to 0.91 (average $=0.62$ ). Observed heterozygosities $\left(H_{\mathrm{O}}\right)$ on average were slightly higher and ranged from 0.17 to 1.0 (average $=0.67$ ). Across-taxa amplification results are presented in Table 2. As expected, these primer pairs tended to amplify Abies taxa known to be more closely related to Fraser fir, especially A. balsamea, but also A. sachalinensis and A. sibirica (Liu 1971; Farjon \& Rushforth 1989). However, less related taxa (A. concolor, A. religiosa, $A$. grandis and $A$. alba) were also successfully amplified with at least seven primer pairs suggesting that these markers may provide useful genetic data for studies aimed at understanding phylogenetic and evolutionary relationships. In addition, the most polymorphic $\left(H_{\mathrm{O}}>c\right.$. 0.5 and $H_{\mathrm{E}}>$ c. 0.5 ) of these markers should prove useful in identifying individual clones within tree breeding and production programs.

The microsatellite marker primer pairs isolated and characterized in this study are being used (i) to assess the genetic structure and genetic diversity of the six major and three minor Fraser fir populations, (ii) to assess whether an association exists between the genetic characteristics of these populations and their size and isolation, and (iii) to assess the evolutionary relationships among Fraser fir and closely related North American fir species within the subgenus Abies subsection Balsameae (Liu 1971). The results will be useful in the development of an ex situ gene conservation plan that could ensure that genetic resources are available for the restoration of extirpated or degraded Fraser fir stands, and for efforts to identify and breed better trees for the economically important Christmas tree industry.

\section{Acknowledgements}

This research was supported (JAB) in part by Dr Judith Williams, University of Southern Mississippi, Department of Biological Sciences, Long Beach, MS.

We also acknowledge the contributions of Dr David O'Malley and Ms. Anne-Margaret Braham, North Carolina State University, for initiating the project and for growing the seedlings used in our analysis, respectively; Dr Ken Jones, Genetic Identification Services, for producing and characterizing the enriched libraries; and Dr Craig Echt and Mr Charles Burdine, Southern Institute of Forest Genetics, for technical contributions. 


\section{References}

Dull CW, Ward JD, Brown HD, Ryan GW, Clerke WH, Uhler RJ (1988) Evaluation of Spruce and Fir Mortality in the Southern Appalachian Mountains. USDA - Forest Service, Southern Region, Atlanta, Georgia, $92 \mathrm{p}$.

Farjon A, Rushforth KD (1989) A classification of Abies Miller (Pinaceae). Notes of the Royal Botanical Garden of Edinburgh, 46, 59-79.

Liu TS (1971) A Monograph of the Genus Abies. Department of Forestry, College of Agriculture, National Taiwan University, Taipei, Taiwan.
Nicholas NS, Eager C, Peine JD (1999) Threatened ecosystem: high-elevation spruce-fir forest. In: Ecosystem Management for Sustainability: Principles and Practices (ed. Peine JD), pp. 431-454. Lewis Publishers, Boca Raton, Florida.

Rozen S, Skaletsky HJ (1996) PRIMER 3. Whitehead Institute for Biomedical Research, Cambridge, Massachusetts.

SAS Institute Inc. (1990) SAS Language Reference, Version 6, 1st edn. SAS Institute Inc., Cary, North Carolina.

Weir BS (1990) GENETIC DATA ANALYSIS. Sinauer Associates, Inc., Sunderland, Massachusetts, $377 \mathrm{p}$. 